\title{
Clinicopathological Analysis of Ultrasound-guided Vacuum-assisted Breast Biopsy for the Diagnosis and Treatment of Breast Disease
}

\author{
HAI-LIN PARK ${ }^{1}$, KA YOUNG KIM ${ }^{2}$, JONG SEOB PARK ${ }^{1}$, JI-EUN SHIN ${ }^{2}$, HYE-RIN KIM ${ }^{2}$, \\ BORA YANG ${ }^{2}$, JI-YOUNG KIM ${ }^{3}$, JEONG YUN SHIM ${ }^{3}$, EUN-AH SHIN ${ }^{3}$ and SONG-MI NOH ${ }^{3}$ \\ ${ }^{1}$ Departments of Surgery, Gangnam CHA Hospital, College of Medicine, CHA University, Seoul, Republic of Korea; \\ ${ }^{2}$ Diagnostic Radiology, Gangnam CHA Hospital, College of Medicine, CHA University, Seoul, Republic of Korea; \\ ${ }^{3}$ Diagnostic Pathology, Gangnam CHA Hospital, College of Medicine, CHA University, Seoul, Republic of Korea
}

\begin{abstract}
Background/Aim: To evaluate the usefulness and safety of vacuum-assisted breast biopsy (VABB) for breast lesion diagnosis and treatment. Patients and Methods: Clinical and histopathological data of 8,748 patients, who underwent 11,221 VABB procedures were analyzed. Results: Most patients (58.2\%) were $<40$ years old. Most lesions $(39.6 \%)$ were $0.6-1.0 \mathrm{~cm}$ in diameter while $3.2 \%$ were $\geq 3.0 \mathrm{~cm}$; fibroadenomas were the most common (46.6\%). Eight (14\% of 57) cases of atypical ductal hyperplasia were underestimated. The positive predictive values (PPVs) of breast imaging reporting and data system (BI-RADS) ultrasound category were $0.6 \%, 3.4 \%, 34.8 \%, 66.2 \%$, and $93.8 \%$ for category $3,4 a, 4 b, 4 c$, and 5 lesions, respectively. The mean number of core specimens was 9.5 \pm 8.8 ; the mean procedure time was $3.4 \pm 2.7 \mathrm{~min}$. No residual lesions were found in $94.4 \%$ of the 7,480 patients. Conclusion: VABB could replace ultrasound-guided core biopsy and surgical excisional biopsy for the diagnosis of breast disease and the treatment of benign breast lesions.
\end{abstract}

Conventional histological methods for identifying breast diseases include fine-needle aspiration cytology (FNAC), core-needle biopsy, and excisional biopsy. FNAC offers the advantages of convenience and cost-effectiveness, but the possibility of obtaining an inadequate specimen is high (3.5$11 \%)$, as is the rate of false negative findings (3-24\%) (1). In contrast, core-needle biopsy has lower rates of obtaining

Correspondence to: Professor Hai Lin Park, Department of Surgery, Gangnam CHA Hospital, CHA University College of Medicine, 569 Nonhyon ro, Seoul 135 080, Republic of Korea. Tel: +82 0234683206, e mail: phl1@cha.ac.kr, parkhl1018@gmail.com

Key Words: Breast disease, vacuum assisted breast biopsy, mammotome excision, ultrasound, underestimation. inadequate specimens and false-negative results (2-4\%), but has the disadvantage of histologic underestimation due to insufficient tissue sampling $(2,3)$. Although excisional biopsy does not have the problems associated with percutaneous biopsy, it leaves a cutaneous scar. Thus, there is a need for new procedures to overcome the limitations of the currently available biopsy methods. Vacuum-assisted breast biopsy (VABB), also known as mammotome biopsy, is gaining greater universal acceptance as a safe and effective procedure that is capable of meeting treatment goals for benign diseases and maximizing diagnostic accuracy by performing excisional biopsy for most breast diseases while minimizing post-procedural scarring and complications.

The present study aimed to investigate the clinical characteristics of patients undergoing VABB and the histopathological characteristics of the biopsied lesions, to determine the efficiency and safety of VABB. To this end, the authors retrospectively analyzed data from 11,221 ultrasound-guided VABB procedures performed in a single center for diagnostic and therapeutic purposes.

\section{Patients and Methods}

In this retrospective study, the authors reviewed the medical records of 8,748 patients who underwent a total of 11,221 ultrasound-guided VABB (Mammotome ${ }^{\circledR}$; Devicor Medical Products, Inc., Cincinnati, $\mathrm{OH}$, USA) procedures at the Department of Surgery, Gangnam CHA Hospital, CHA University of Medicine, over a 13-year period (January 2003 to December 2015), together with the histopathological findings of each biopsied lesion. Complete excisional VABB was performed for Breast Imaging Reporting and Data System (BI-RADS) ultrasound category 3 or 4 a lesions, whereas incisional biopsy was performed for category $4 \mathrm{~b}-5$ lesions. Category 3 lesions were followed up by conventional methods with 6-monthly ultrasound examinations. During this follow-up period, $\mathrm{VABB}$ was performed if the long and short axes of the lesion increased to $>3 \mathrm{~mm}$ or if the shape of the lesion upgraded to category $4 \mathrm{a}$ or higher. Other criteria for performing VABB on 
category 3 lesions included difficulty in instituting per-protocol follow-up (such as geographic constraints); planned pregnancy or mammoplasty; extreme patient anxiety due to palpable lesions; history of ipsilateral breast cancer surgery; family or personal history of breast cancer; and FNAC or core-needle biopsy findings that were non-definitive or showed borderline or B3 lesions, i.e. lesions showing uncertain malignant potential such as papillary neoplasia and atypical ductal hyperplasia (ADH), phyllodes tumors, and mucinous lesions.

An 8-gauge needle was used for VABB of all lesions except those in women who had prosthetic breast implants. All procedures were performed by a single surgeon in an operating room, under local anesthesia. A 3-5-mm skin incision was made and the procedure was continued until complete removal of the lesion was confirmed by ultrasonography. The procedure time was measured from the time that ultrasound-guided target lesion localization was initiated to the time the probe was removed from the breast after achieving complete excision of the target lesion.

Breast ultra-sonograms were obtained using duplex ultrasonography with B mode and color Doppler with a high-frequency (7-12 MHz) linear array probe (Logic 700; GE Healthcare Bio sciences, Pittsburgh, PA, USA; HDI 5000; Philips Ultrasound, Bothell, WA, USA). During VABB, ultrasonography was performed using duplex sonography with B-mode with a high-frequency (7-12 MHz) linear array probe (Ecube: Alpinion healthcare, Seoul, Republic of Korea). Tumor size was determined based on the longest dimension, but the long cytoplasmic processes of the tumor were excluded from the measurement. Sagittal and transverse views were obtained for each lesion, and the longest dimension was obtained for each transducer position. Whenever possible, the longest dimension was obtained collinear to the ultrasound beam.

The present study was performed with the approval of the Institutional Ethical Committee of Gangnam CHA University Hospital (approval number, KNC13 016).

Statistical analysis. Statistical analyses were performed using SPSS for Windows, version 18.0 (SPSS, Chicago, IL, USA). The Chisquare test was used to analyze the rate of malignancy according to BI-RADS ultra-sonographic category, and to compare the proportion of invasive versus noninvasive cancers according to ultra-sonographic category. The authors evaluated efficiency by determining the complete excision rate and safety by assessing the complication rate. Differences between groups were evaluated using the Chi-square test, simple linear regression, or ANOVA, as appropriate. A $p$-value of 0.05 or less was considered statistically significant.

\section{Results}

Characteristics of the patients and the lesions. The patients' mean age was 37.8 years, and $58.2 \%(n=5,091)$ were aged $<40$ years (Table I). Most lesions ( $n=4,443$ [39.6\%]) were $0.6-1.0 \mathrm{~cm}$ in size; the largest lesion was a fibroadenoma measuring $7.9 \mathrm{~cm}$. A total of 1,276 (11.4\%) lesions were defined as being relatively large, measuring $\geq 2.1 \mathrm{~cm}$. Of these, a significantly greater proportion occurred in women aged $<40$ years than in women aged $\geq 40$ years $(67.2 \%$ $[\mathrm{n}=857]$ vs. $32.8 \% \quad[\mathrm{n}=419]$, respectively; $p<0.001)$. Moreover, $130(10.2 \%)$ of the relatively large lesions were malignant, requiring subsequent breast cancer surgery. The rate of malignancy increased with age, being $0 \%, 1.7 \%$, $8.6 \%, 16.7 \%$, and $28.7 \%$ in those aged 10-19, 20-29, 30-39, $40-49$, and $\geq 50$ years, respectively $(p<0.001)$. On physical examination, 3,524 (31.4\%) lesions were palpable and 7,697 $(68.6 \%)$ were non-palpable. A fairly high proportion $(43.3 \%$ $[\mathrm{n}=553])$ of larger tumors $(\geq 2.1 \mathrm{~cm}$ in size) were nonpalpable (Tables I and II).

Histopathological characteristics of the lesions. Fibroadenoma was the most common histopathological type of benign breast tumor, accounting for $46.6 \%(n=5,226)$ of cases. Malignancy was confirmed in $414(3.7 \%)$ cases; these patients underwent additional cancer surgery (Table III).

Comparison of BI-RADS ultrasound category and histologic diagnosis. Of the $6,791(60.5 \%)$ category 3 lesions, 42 were confirmed as malignant by histopathological evaluation; hence, the positive predictive value (PPV) was $0.6 \%$. Thirteen $(31.0 \%)$ of these were invasive cancers, including $10(23.9 \%)$ cases of invasive ductal carcinoma and three $(7.1 \%)$ of micropapillary carcinoma. Twenty-six (61.9\%) - including 18 (42.9\%) cases of ductal carcinoma in situ - were noninvasive cancers (Table IV). Of the 4,019 (35.8\%) category 4a lesions, $138(\mathrm{PPV}=3.4 \%)$ were confirmed as malignant by histopathological evaluation. Of these, 59 (42.8\%) were invasive cancers and $77(55.8 \%)$ were noninvasive cancers, including $60(43.5 \%)$ cases of ductal carcinoma in situ. Of the $221(2.0 \%)$ category $4 \mathrm{~b}$ lesions, $77(\mathrm{PPV}=34.8 \%)$ were malignant; invasive cancer accounted for $68.8 \%(n=53)$ and noninvasive cancer for $31.2 \%(n=24)$. Of the $77(0.7 \%)$ category 4c lesions, $51(\mathrm{PPV}=66.2 \%)$ were found to be malignant on histopathological evaluation; invasive cancer accounted for $96.1 \%(n=49)$, and two $(3.9 \%)$ cases of lobular carcinoma in situ were identified as being noninvasive cancers. Of the $113(2.0 \%)$ category 5 lesions, $106(93.8 \%)$ were confirmed as malignant by histopathological evaluation: invasive cancer accounted for $86.8 \%(n=92)$ and $14(13.2 \%)$ were non-invasive (ductal carcinoma in situ). There was a significant association between assessments made by breast ultrasonography and the VABB biopsy results $(p<0.001)$.

The malignancy rate did not differ significantly between category 3 and 4 a lesions $(p=0.261)$ or between category $4 c$ and 5 lesions $(p=0.072)$, but there were significant differences between category $4 \mathrm{a}$ and $4 \mathrm{~b}(p=0.001)$ and category $4 \mathrm{~b}$ and $4 c(p<0.001)$ lesions. Moreover, the proportion of invasive cancers increased significantly with incremental increases in BI-RADS ultrasound category $(p<0.05)$.

Lesion size, number of core pieces removed, and VABB procedure time. For all completely excised lesions, the mean ( \pm standard deviation) number of core specimens removed was $9.5 \pm 8.8$ and the mean procedure time was $3.4 \pm 2.7 \mathrm{~min}$ (Table V). 
Table I. Clinical features of patients who underwent vacuum-assisted breast biopsy.

\begin{tabular}{lcc}
\hline Variable & Category & $\mathrm{n}(\%)$ \\
\hline Age, years $(\mathrm{n}=8,748)$ & $10-19$ & $68(0.8)$ \\
& $20-29$ & $1575(18.0)$ \\
& $30-39$ & $3448(39.4)$ \\
& $40-49$ & $2644(30.2)$ \\
Lesion size, cm $(\mathrm{n}=11,221)$ & $50-59$ & $830(9.5)$ \\
& $>60$ & $183(2.1)$ \\
& $\leq 0.5$ & $993(8.8)$ \\
& $0.6-1.0$ & $4443(39.6)$ \\
Physical examination $(\mathrm{n}=11,221)$ & $1.1-1.5$ & $3141(28.0)$ \\
& $1.6-2.0$ & $1368(12.2)$ \\
Location $(\mathrm{n}=11,221)$ & $2.1-3.0$ & $921 /(8.2)$ \\
& $>3.0$ & $355(3.2)$ \\
& Palpable lesions & $3524(31.4)$ \\
Multiplicity $(\mathrm{n}=11,221)$ & Non-palpable lesions & $7697(68.6)$ \\
& Right & $3786(43.2)$ \\
& Left & $3869(44.2)$ \\
& Bilateral & $1093(12.6)$ \\
& Multiple lesions & $8778(78.2)$ \\
& Single lesion & $2443(21.8)$ \\
& &
\end{tabular}

\section{Complications}

Hemorrhage. One patient - a 22-year-old woman with a 2.5$\mathrm{cm}$ benign tumor located in accessory breast tissue in the left axilla - experienced massive bleeding during the VABB procedure as the axillary vein was penetrated by the tip of the 8-gauge probe. The patient required a blood transfusion (of approximately 1,000 ml). Axillary exploration was performed under general anesthesia; the bleeding focus was identified and successfully managed. The patient had mild to moderate hematomas in the breast after the procedure. Apart from this case, no other serious or critical complications (such as intraprocedural hemorrhage requiring blood transfusion and conversion to open surgery) occurred. For all other patients with bleeding in the breast, manual compression was the best treatment option; no other case required open exploration to find the site of bleeding.

Vasovagal reflex. Seven patients complained of vasovagal reflex symptoms after administration of a $1 \%$ lidocaine injection. The main symptoms were bradycardia, dizziness, hypotension, nausea/vomiting, and dyspnea.

Underestimation of lesions. Inaccurate diagnosis, such as histologic underestimation, was only seen in cases of $\mathrm{ADH}$. In this study, the lesions in 57 cases were identified as ADH. Of these, very small lesions (observed in only one focus per 10 high-power fields) were classified as focal ADH and were followed up accordingly. Other lesions, observed in two or more foci per high-power field, were classified as $\mathrm{ADH}$ and
Table II. Ultra-sonographic features of the biopsied lesions according to BI-RADS ultrasound category $(n=11,221)$.

\begin{tabular}{lc}
\hline BI-RADS ultrasound category & $\mathrm{n}(\%)$ \\
\hline Category 3 & $6,791(60.5)$ \\
Category 4a & $4,019(35.8)$ \\
Category 4b & $221(2.0)$ \\
Category 4c & $77(0.7)$ \\
Category 5 & $113(1.0)$ \\
\hline
\end{tabular}

BI-RADS: Breast imaging reporting and data system.

Table III. Pathologic classification of breast lesions removed by VABB $(n=11,221)$.

\begin{tabular}{lc}
\hline Pathologic classification & $\mathrm{n}(\%)$ \\
\hline Fibroadenoma & $5,226(46.6)$ \\
Fibrocystic disease & $2,732(24.3)$ \\
Malignancy & $414(3.7)$ \\
Intraductal papilloma & $403(3.6)$ \\
Ductal epithelial hyperplasia & $260(2.3)$ \\
Sclerosing adenosis & $155(1.4)$ \\
Phyllodes tumor (benign, borderline) & $93(0.8)$ \\
Atypical ductal epithelial hyperplasia & $57(0.5)$ \\
Other benign diseases & $1,881(16.8)$ \\
\hline
\end{tabular}

VABB: Vacuum-assisted breast biopsy.

additional surgical biopsy was performed. Eleven patients underwent additional surgical biopsy. Of these, eight had lesions that were upgraded to malignancy. Among those who did not undergo an additional excision biopsy, none showed signs of recurrence or regrowth on follow-up ultrasonography or mammography during the follow-up period. Therefore, of the 57 cases of ADH that underwent complete excisional biopsy using ultrasound-guided VABB, histologic underestimation was observed in eight $(14.0 \%)$ cases: three were low grade ductal carcinoma in situ, three were intermediate-grade ductal carcinoma in situ, and two were multifocal lobular carcinoma in situ.

Breast implant injury or infection. Overall, $258(2.3 \%)$ patients had undergone aesthetic breast surgery and had implants in situ. One case of implant rupture occurred during the VABB procedure - the implant had to be removed. No cases of implant infection occurred.

Incomplete excision of the target lesion. Follow-up ultrasonography was performed 3-6 months after the procedure to check if complete excision of all target tissue had been achieved and to confirm the absence of residual lesions. Of the 11,221 cases, only 7,480 were followed up 
Table IV. Comparison of the histopathological classification and BI-RADS ultrasound category of lesions removed by VABB.

\begin{tabular}{|c|c|c|c|c|c|}
\hline \multirow[t]{2}{*}{ Histopathological classification } & \multicolumn{5}{|c|}{ BI-RADS ultrasound category, n (\%) } \\
\hline & $\begin{array}{c}3 \\
(\mathrm{n}=6791)\end{array}$ & $\begin{array}{c}4 \mathrm{a} \\
(\mathrm{n}=4019)\end{array}$ & $\begin{array}{c}4 b \\
(n=221)\end{array}$ & $\begin{array}{c}4 c \\
(n=77)\end{array}$ & $\begin{array}{c}5 \\
(\mathrm{n}=113)\end{array}$ \\
\hline Noninvasive cancer & $26(61.9)$ & $77(55.8)$ & $24(31.2)$ & $2(3.9)$ & $14(13.2)$ \\
\hline Ductal carcinoma in situ & $18(42.9)$ & $60(43.5)$ & $18(23.4)$ & 0 & $14(13.2)$ \\
\hline Lobular carcinoma in situ & $5(11.9)$ & $9(6.5)$ & $2(2.6)$ & $2(3.9)$ & \\
\hline Intraductal papillary carcinoma & $3(7.1)$ & $8(5.8)$ & $4(5.2)$ & 0 & \\
\hline Invasive cancer & $13(31.0)$ & $59(42.8)$ & $53(68.8)$ & $49(96.1)$ & $92(86.8)$ \\
\hline Invasive ductal carcinoma & $10(23.9)$ & $48(34.8)$ & $49(63.6)$ & $45(88.2)$ & $86(81.1)$ \\
\hline Micropapillary carcinoma & $3(7.1)$ & 0 & 0 & 0 & \\
\hline Mucinous carcinoma & 0 & $4(2.9)$ & $2(2.6)$ & 0 & \\
\hline Solid papillary carcinoma & 0 & $5(3.6)$ & 0 & 0 & \\
\hline Secretory carcinoma & 0 & $1(0.7)$ & 0 & $2(3.9)$ & \\
\hline Medullary carcinoma & 0 & $1(0.7)$ & 0 & 0 & \\
\hline Focal lobular carcinoma & 0 & 0 & $1(1.3)$ & 0 & \\
\hline Apocrine ductal carcinoma & 0 & 0 & $1(1.3)$ & 0 & \\
\hline Tubular carcinoma & 0 & 0 & 0 & $2(3.9)$ & $1(0.9)$ \\
\hline Invasive lobular carcinoma & 0 & 0 & 0 & 0 & $3(2.9)$ \\
\hline Invasive cribriform carcinoma & 0 & 0 & 0 & 0 & $2(1.9)$ \\
\hline Malignant phyllodes tumor & $3(7.1)$ & $2(1.4)$ & 0 & 0 & 0 \\
\hline Malignant lesions, n (PPV) & $42(0.6)$ & $138(3.4)$ & $77(34.8)$ & $51(66.2)$ & $106(93.8)$ \\
\hline
\end{tabular}

BI-RADS: Breast imaging reporting and data system; VABB: vacuum-assisted breast biopsy; PPV: positive predictive value.

after the procedure; no signs of residual lesions were found in $7,060(94.4 \%)$ of those followed up. Of the 420 cases where residual lesions were found on follow-up ultrasonography, only four underwent additional VABB to remove tumors that had regrown. In all four cases, the lesions were found to be benign. The remaining cases are still being followed-up; none have shown signs of tumor regrowth or malignant transformation.

\section{Discussion}

The incidence of breast cancer has steadily increased in South Korea as well as in many Western countries. Young premenopausal women in Asian countries, including Korea, have a higher incidence of breast cancer than their Western counterparts; this has increased the level of interest in screening for early-stage breast cancer.

Recent advances in ultrasonography have allowed early detection of non-palpable malignant lesions smaller than 1 $\mathrm{cm}$, contributing to the increase in breast cancer survival rates. Although most surgeons still opt for traditional excision biopsy of palpable lesions, this method is expected to be used increasingly less frequently. Worldwide, coreneedle biopsy is the most commonly used method for both non-palpable and palpable lesions, given its high accuracy, cost effectiveness, low complication rate (hemorrhage or infection), and convenience. Despite these advantages, it is
Table V. Lesion size, number of core pieces removed, and VABB procedure time.

\begin{tabular}{lcc}
\hline Lesion size $(\mathrm{cm})$ & $\begin{array}{c}\text { Number of core pieces } \\
\text { (8-gauge), mean } \pm \text { SD }\end{array}$ & $\begin{array}{c}\text { Procedure time } \\
(\min ), \text { mean } \pm \text { SD }\end{array}$ \\
\hline $0.1-0.5(\mathrm{n}=993)$ & $6.2 \pm 3.7$ & $3.2 \pm 2.6$ \\
$0.6-1.0(\mathrm{n}=4443)$ & $6.9 \pm 3.8$ & $3.0 \pm 1.8$ \\
$1.1-1.5(\mathrm{n}=3141)$ & $8.8 \pm 4.7$ & $3.1 \pm 2.1$ \\
$1.6-2.0(\mathrm{n}=1368)$ & $11.7 \pm 8.0$ & $3.8 \pm 3.1$ \\
$2.1-3.0(\mathrm{n}=921)$ & $18.0 \pm 12.6$ & $4.6 \pm 3.7$ \\
$>3.0(\mathrm{n}=355)$ & $26.3 \pm 26.8$ & $6.0 \pm 5.9$ \\
Total $(\mathrm{n}=11221)$ & $9.5 \pm 8.8$ & $3.4 \pm 2.7$ \\
\hline
\end{tabular}

VABB: Vacuum-assisted breast biopsy; SD: standard deviation.

not a fail-proof method due to its inherent drawbacks, namely, high rates of false-negative findings and misclassification (underestimation of disease), which are inevitable because the procedure samples only parts of the target lesion, not the entire lesion. Morris et al. reported that $29 \%$ of breast lesions are heterogeneous, yielding different histologic results from the center and periphery; thus, targeting one part of a heterogeneous mass could lead to misdiagnosis (2). Moreover, Joshi et al. reported that core biopsy findings of $\mathrm{ADH}$ underestimate the diagnosis of malignancy by $18-88 \%$ (3). 
Carder et al. performed additional excision biopsies on 26 patients who were diagnosed with B3 lesions (uncertain malignant potential) on core needle biopsy specimens. Although 22 had benign pathology at surgical excision, four had malignant lesions. According to the authors of that study, the difficulty in establishing a histologic diagnosis from specimens obtained via core needle biopsy stems largely from the limited amount of tissue obtained and uncertainties over whether the tissue is representative. They maintained that newer vacuum-assisted techniques, such as the mammotome, provide larger tissue samples and may help to reduce the rate of misclassification of tumors as being benign (4). VABB, developed to overcome the shortcomings of percutaneous biopsy, is now widely used because it allows complete removal of the target lesion and guarantees high diagnostic accuracy and therapeutic results (5).

The main indications for VABB at our institution are palpable or non-palpable BI-RADS ultrasound category 3 or 4a lesions, for which complete excisional VABB is usually performed. Category $4 \mathrm{~b}-5$ lesions are generally subjected to core biopsy, but incisional biopsy with VABB may be performed to acquire a larger sample for diagnostic purposes in patients on neoadjuvant chemotherapy who require a more accurate histologic and molecular biological diagnosis $(5,6)$. No consensus has yet been reached on how to deal with USG BI-RADS category 3 lesions, which are commonly found on ultrasonography. The conventional management of category 3 lesions, which have a malignancy rate of $0.5-2 \%$, is 3-6monthly follow-up with ultrasonography; this is less invasive and more cost-effective than percutaneous core biopsy. Some reports suggest that the triple test can differentiate between benign and malignant lesions with an accuracy of approximately $95 \%$, and routine follow-up for 1-3 years to monitor changes in the features of the lesion is recommended (6-8). Proponents of this conservative approach argue that most fibroadenomas, which make up the vast majority of breast benign lesions, have already stopped growing at the time of their discovery, and even if they grow further, such growth generally stops at $2-3 \mathrm{~cm}$ in diameter. In fact, the lesions decrease in size in $15 \%$ of cases, and continued growth occurs in only $5-10 \%$ of all cases. Moreover, progression of a fibroadenoma to breast cancer is extremely rare (6-9).

Even though follow-up at 6-monthly intervals is recommended in most patients with category 3 lesions, the authors recommend VABB as an option for the following patients: i) those with a low probability of attending regular follow-up, ii) those planning a pregnancy or breast augmentation surgery, iii) those with extreme anxiety related to the lesion, iv) those with a lesion that increases in size during the course of follow-up, v) those with subjective symptoms or pain with BI-RADS ultrasound category 3-4 lesions, vi) those with a palpable mass, vii) those with a personal or family history of breast cancer, viii) those with BI-
RADS ultrasound category 3 lesions planning conservative surgery in the ipsilateral breast, and ix) those with suspected multifocal or multicentric breast cancer $(10,11)$.

If the option of biopsy is chosen for category 3 or 4 lesions, one should decide whether to use VABB or core needle biopsy using an automated biopsy gun. Parker recommends excision via $\mathrm{VABB}$ for lesions $<1.5 \mathrm{~cm}$ and conventional core needle biopsy for larger lesions, because accurate targeting is crucial for small lesions, especially those $<1 \mathrm{~cm}$; this is a great challenge when using a biopsy gun (11). Even if the surgeon succeeds in obtaining five tissue samples or more, it is often difficult to confirm whether tissue from the area of interest has been sampled. Such uncertainty increases if the biopsy results point to nonspecific benign disease and if the biopsy results contradict the ultrasonographic findings; this may prompt the surgeon to perform additional surgical excision. The problem of uncertainty can be resolved by completely removing the lesion via ultrasound-guided VABB (12).

In this study, the majority of patients (58.2\%) were women aged <39 years, a considerably higher proportion than those reported in studies by Kim D et al. (13) and Kim SH et al. (14), of $37.2 \%$ and $35.2 \%$, respectively. Various factors may be behind the different results found in studies from Korea, such as lower childbirth rates associated with delayed marriage, higher rates of smoking and alcohol consumption, and higher workplace stress. More importantly, however, it may be attributable to the increased interest and more active participation in breast self-examination and ultrasound screening by Korean women in their 20s and 30s, spurred on by the increased incidence of breast cancer among premenopausal women in Korea.

In this study, fibroadenoma was the most common histopathological type of benign breast tumor, accounting for $46.6 \%(n=5,226)$ of cases. It is still debatable whether fibroadenomas are likely to develop into breast cancer. Based on long-term follow-up results, many studies have reported that patients with biopsy-confirmed fibroadenomas have a high relative risk of developing breast cancer (15-18). In a study by Carter et al., among 685 patients with fibroadenoma followed up for 8.3 years, the relative risk of breast cancer was 1.7 (16). Thus, breast specialists should recommend active follow-up of patients with histologically confirmed fibroadenomas. Moreover, if a breast tumor is discovered in a patient with a growing fibroadenoma or with a family history of breast cancer, a more aggressive follow-up approach should be adopted (such as removing the tumor or performing a biopsy), rather than opting for routine follow-up.

In the current study, it was observed that patients commonly presented with multiple lesions $(78.2 \%)$, and that most lesions $(\mathrm{n}=4,443[39.6 \%])$ were $0.6-1.0 \mathrm{~cm}$ in size. The largest lesion was a fibroadenoma measuring $7.9 \mathrm{~cm}$. Surgical excision biopsy was recommended for this patient, but she refused this 
option as she had a tendency to develop hypertrophic scarring. She thus underwent successful excisional VABB with no complications or residual lesion. The procedure time was approximately $15 \mathrm{~min}$, and 97 core pieces were removed.

In this study, 1,276 (11.4\%) lesions were defined as being relatively large $(>2.1 \mathrm{~cm}$ in long-axis diameter on ultrasonography). Approximately two-third of the lesions occurred in women younger than 40 years, and $10.2 \%$ were found to be malignant. The rate of malignancy increased with age. In other words, whereas the rate of having relatively large tumors was higher among women aged $<40$ years than $\geq 40$ years, the probability of relatively large tumors being malignant tended to increase with increasing age. There is no clear explanation for the high prevalence of breast tumors among young women aged 20-39 years, but it is suspected that this may be attributable to physical growth and increasing obesity rates due to Westernized diet and lifestyle, as well as excessive alcohol consumption (19-21). A further finding was that approximately $43.3 \%$ of relatively large tumors were non-palpable, emphasizing the need for greater awareness of the necessity to perform regular ultrasonographic breast examinations among women younger than 40 years. Although no comparative analysis can be made due to lack of research reports on positive correlations between tumor size (measured by ultrasound) and malignancy rate, our findings suggest the need to biopsy relatively large tumors found on ultrasound examination in patients aged $\geq 40$ years to determine whether such lesions are malignant.

According to the first edition of BI-RADS, ultrasound category 3 lesions typically have a malignancy rate of $0.5-2 \%$, whereas the overall positive predictive values for categories 4 and 5 are 3-94\% and $>95 \%$, respectively (22). In this study, the PPVs for BI-RADS ultrasound categories $3,4 \mathrm{a}, 4 \mathrm{~b}, 4 \mathrm{c}$, and 5 were $0.6 \%, 3.4 \%, 34.8 \%, 66.2 \%$, and $93.8 \%$, respectively. These findings are fairly consistent with results from previous studies. Wiratkapun et al. reported that $94 \%$ of 497 patients with category 5 lesions were diagnosed with breast cancer and that categories $4 \mathrm{a}, 4 \mathrm{~b}$, and $4 \mathrm{c}$ showed PPVs of 9\%, 21\%, and 57\%, respectively (23). Yoon et al. reported PPVs of $7.6 \%$ (149/1963), 37.8\% (68/180), and $81.9 \%(235 / 287)$ for category $4 \mathrm{a}, 4 \mathrm{~b}$, and $4 \mathrm{c}$ lesions, respectively (24). These results, obtained from different research institutions, are all very similar. Furthermore, the ratio of invasive to noninvasive carcinomas were $31.0 \%$, $42.8 \%, 68.8 \%, 96.1 \%$, and $86.8 \%$ for BI-RADS ultrasound categories 3, 4a, 4b, 4c, and 5, respectively. Linear regression analysis demonstrated an increasing trend in the ratio of invasive to non-invasive carcinoma with an increasing level of BI-RADS ultrasound category $(p<0.05)$.

These results may be attributable to the BI-RADS ultrasound classification system used. Various studies, such as those conducted by Kim EK et al. (25) and Jales et al. (26), have reported that suspicious ultrasound findings can be divided into major and minor suspicious findings: Major suspicious findings include an irregular shape, spiculated margin, and microcalcifications, whereas minor suspicious findings include a microlobulated or angular margin, nonparallel orientation, duct extension, complex echogenicity, and posterior shadowing. Accordingly, the categories were recategorized as category $4 \mathrm{a}$ (one or more minor suspicious findings), category $4 \mathrm{~b}$ (more than three minor suspicious findings), category $4 \mathrm{c}$ (one major suspicious feature with or without minor suspicious features), and category 5 (two or more major suspicious findings). Since higher category levels include major findings such as spiculated margin and/or irregular shape, the proportion of invasive carcinomas rather than in situ lesions would increase.

In terms of the number of core specimens required and VABB procedure time, from our findings it is inferred that to successfully achieve complete excision of lesions $<2.0 \mathrm{~cm}$ in size using an 8 -gauge probe, at least 12 core samples are needed, while an average of 18 samples are needed for lesions $<3 \mathrm{~cm}$ in size. Although no comparative analysis can be made on the number of cores removed or the procedure time due to the lack of related research data, the results of this study demonstrate that the mean procedure time is close to three minutes, which is considerably shorter than the time required for conventional surgical excision biopsy. Even if an additional 5-10 min is required to apply pressure to stop bleeding and it may take longer than 3 minutes for less experienced surgeons, the time-saving benefit of VABB remains clear.

To date, several complications have been reported with the use of VABB. However, in this study, there were very few complications, such as damage to breast implants; severe bleeding requiring conversion to open surgery; and misclassification (histological underestimation), except in cases of ADH. Regarding the success rate of VABB, complete removal was confirmed in $94.4 \%$ of all cases followed-up; residual lesions were identified in the remaining $5.6 \%$ of cases. For patients with malignant tumors, residual lesions were not problematic because additional breast cancer surgery was performed. In patients with benign tumors, the residual lesion typically did not require excision surgery. However, because the residual lesion can regrow, follow-up ultrasound examination is required. In this study, four patients required a repeat $\mathrm{VABB}$ procedure because regrowth was detected during the follow-up period - the lesions in all four cases were confirmed to be benign.

Many studies have reported on histologic underestimation of cases of $\mathrm{ADH}$ diagnosed by ultrasound-guided VABB. Grady et al. reported that the rate of $\mathrm{ADH}$ underestimation was $33 \%$ (6 of 18) when incisional VABB was performed and $0 \%$ (0 of 29) when complete ultrasound-guided, percutaneous excision was performed, a result equivalent to open surgical biopsy. They stated that complete ultrasound-guided, vacuumassisted, percutaneous excision is more accurate than non- 
excisional ultrasound-guided biopsy, and that patients so diagnosed have very low underestimation rates and may not require open surgical re-excision (27). In this study, eight (14.0\%) of the 57 cases of ADH that were diagnosed after complete excision biopsy using ultrasound-guided VABB had histological underestimation. Hence, patients diagnosed with ADH by VABB should undergo additional surgical biopsy if deemed necessary. Moreover, further research is required to determine the indications for additional surgical biopsy in patients with $\mathrm{ADH}$ diagnosed on VABB specimens.

This was a retrospective study of the effectiveness ultrasound-guided VABB in Korean patients, the clinical profile of such patients, and the histopathological profile of the biopsied lesions. The study demonstrated a high incidence of tumors in young Korean women, unlike in the West. However, the PPV of VABB, based on BI-RADS ultrasound category, was very similar to those reported in previous Western studies. The authors also examined how many core specimens need to be removed for lesions of different sizes to achieve complete excision of the target lesion using 8-gauge probe VABB; these results are considered to be especially meaningful. However, this study also had some limitations. Because the sample size was too large, it was not possible to conduct an analysis by size and age in patients with residual lesions. The authors were also unable to investigate the incidence of hematoma and minor bleeding, which were the most common complications.

In conclusion, VABB excision of breast lesions is safe and useful for both diagnostic and therapeutic purposes. Its use is associated with minimal serious adverse events such as severe bleeding, histological underestimation, and incomplete removal of target lesions. It overcomes the drawbacks of ultrasound-guided core biopsy and surgical excision biopsy methods, that is expected to completely replace. The participants of this study are still being followed-up with ultrasound examinations at 6-monthly intervals, and the authors plan to report the long-term follow-up findings.

\section{Conflicts of Interest}

The Authors declare that they have no competing interests.

\section{References}

1 Fornage BD, Faroux MJ and Simatos A: Breast masses: USguided fine-needle aspiration biopsy. Radiology 162: 409-414, 1987.

2 Morris EA, Liberman L, Trevisan SG, Abramson AF and Dershaw DD: Histologic heterogeneity of masses at percutaneous breast biopsy. Breast J 8: 187-191, 2002.

3 Joshi M, Duva-Frissora A, Padmanabhan R, Greeley J, Ranjan A, Ferrucci F, Kwon J and Khettry U: Atypical ductal hyperplasia in stereotactic breast biopsies: enhanced accuracy of diagnosis with the mammotome. Breast J 7: 207-213, 2001.
4 Carder PJ and Liston JC: Will the spectrum of lesions prompting a "B3" breast core biopsy increase the benign biopsy rate? J Clin Pathol 56: 133-138, 2003.

5 Park HL and Kim LS: The current role of vacuum assisted breast biopsy system in breast disease. J Breast Cancer 14: 1-7, 2011.

6 Cant PJ, Madden MV, Coleman MG and Dent DM: Nonoperative management of breast masses diagnosed as fibroadenoma. Br J Surg 82: 792-794, 1995.

7 Greenberg R, Skornick Y and Kaplan O: Management of fibroadenoma. J Gen Intern Med 13: 640-643, 1998.

8 Cant PJ, Madden MV, Colse PM, Learmonth GM, Hacking EA and Dent DM: Case for conservative management of selected fibroadenomas of the breast. Br J Surg 74: 857-859, 1987.

9 Alle KM, Moss J, Venegas RJ, Khalkhali I and Klein SR: Conservative management of fibroadenoma of the breast. Br J Surg 83: 992-993, 1996.

10 Baez E, Huber A, Vetter M and Hackeloer BJ: Minimal invasive complete excision of benign breast tumors using a threedimensional ultrasound-guided mammotome vacuum device. Ultrasound Obstet Gynecol 21: 267-272, 2003.

11 Parker SH, Klaus AJ, McWey PJ, Schilling KJ, Cupples TE, Duchesne N, Guenin MA and Harness JK: Sonographically guided directional vacuum assisted breast biopsy using a handheld device. Am J Roentgenol 177: 405-408, 2001.

12 Park HL, Kwak JY, Lee SH, Kim JY, Kim KI, Kim WW, Han SM and Lee KS: Excision of benign breast disease by ultrasoundguided vacuum assisted biopsy device (Mammotome). Ann Surg Treat Res 68: 96-101, 2005.

13 Kim D, Lee BC, Jang SY, Ryu JK, Park SY, Kim HJ, Kim L and Yoon SO: Usefulness of ultrasound guided vacuum-assisted mammotome biopsy for breast lesion. Ann Surg Treat Res 64: 109-114, 2003.

14 Kim SH, Choi JW, Ko EY, Lee KS, Min SK, Kim IG, Yang DH, Lee BH and Kim LS: Minimally invasive excision of benign breast tumor using ultrasound guided vacuum-assisted Mammotome. Ann Surg Treat Res 65: 279-283, 2003.

15 Dyrstad SW, Yan Y, Fowler AM and Colditz GA: Breast cancer risk associated with benign breast disease: systematic review and meta-analysis. Breast Cancer Res Ttreat 149: 569-575, 2015.

16 Carter CL, Corle DK, Micozzi MS, Schatzkin A and Taylor PR: A prospective study of the development of breast cancer in 16,692 women with benign breast disease. Am J Epidemiol 128: 467-477, 1988.

17 Levi F, Randimbison L, Te VC and La Vecchia C: Incidence of breast cancer in women with fibroadenoma. Int J Cancer 57: 681-683, 1994.

18 Dupont WD, Page DL, Parl FF, Vnencak-Jones CL, Plummer WD Jr., Rados MS and Schuyler PA: Long-term risk of breast cancer in women with fibroadenoma. New Engl J Med 331: 10$15,1994$.

19 Strong AL, Strong TA, Rhodes LV, Semon JA, Zhang X, Shi Z, Zhang S, Gimble JM, Burow ME and Bunnell BA: Obesity associated alterations in the biology of adipose stem cells mediate enhanced tumorigenesis by estrogen dependent pathways. Breast Cancer Res 15: R102, 2013.

20 Roswall $\mathrm{N}$ and Weiderpass E: Alcohol as a risk factor for cancer: existing evidence in a global perspective. J Prev Med Public Health 48: 1-9, 2015. 
21 Hirko KA, Chen WY, Willett WC, Rosner BA, Hankinson SE, Beck AH, Tamimi RM and Eliassen AH: Alcohol consumption and risk of breast cancer by molecular subtype: Prospective analysis of the nurses' health study after 26 years of follow-up. Int J Cancer 138: 1094-1101, 2016.

22 Liberman L and Menell JH: Breast imaging reporting and data system (BI-RADS). Radiol Clin North Am 40: 409-430, 2002.

23 Wiratkapun C, Lertsithichai P and Wibulpholprasert B: Positive predictive value of breast cancer in the lesions categorized as BIRADS category 5. J Med Assoc Thailand 89: 1253-1259, 2006.

24 Yoon JH, Kim MJ, Moon HJ, Kwak JY and Kim EK: Subcategorization of ultrasonographic BI-RADS category 4: positive predictive value and clinical factors affecting it. Ultrasound Med Biol 37: 693-699, 2011.

25 Kim EK, Ko KH, Oh KK, Kwak JY, You JK, Kim MJ and Park BW: Clinical application of the BI-RADS final assessment to breast sonography in conjunction with mammography. AJR Am J Roentgenol 190: 1209-1215, 2008.
26 Jales RM, Sarian LO, Torresan R, Marussi EF, Alvares BR and Derchain S: Simple rules for ultrasonographic subcategorization of BI-RADS(R)-US 4 breast masses. Eur J Radiol 82: 12311235, 2013.

27 Grady I, Gorsuch H and Wilburn-Bailey S: Ultrasound-guided, vacuum-assisted, percutaneous excision of breast lesions: an accurate technique in the diagnosis of ADH. J Am Coll Surg 201: 14-17, 2005.
Received February 8, 2018

Revised February 28, 2018

Accepted March 1, 2018 